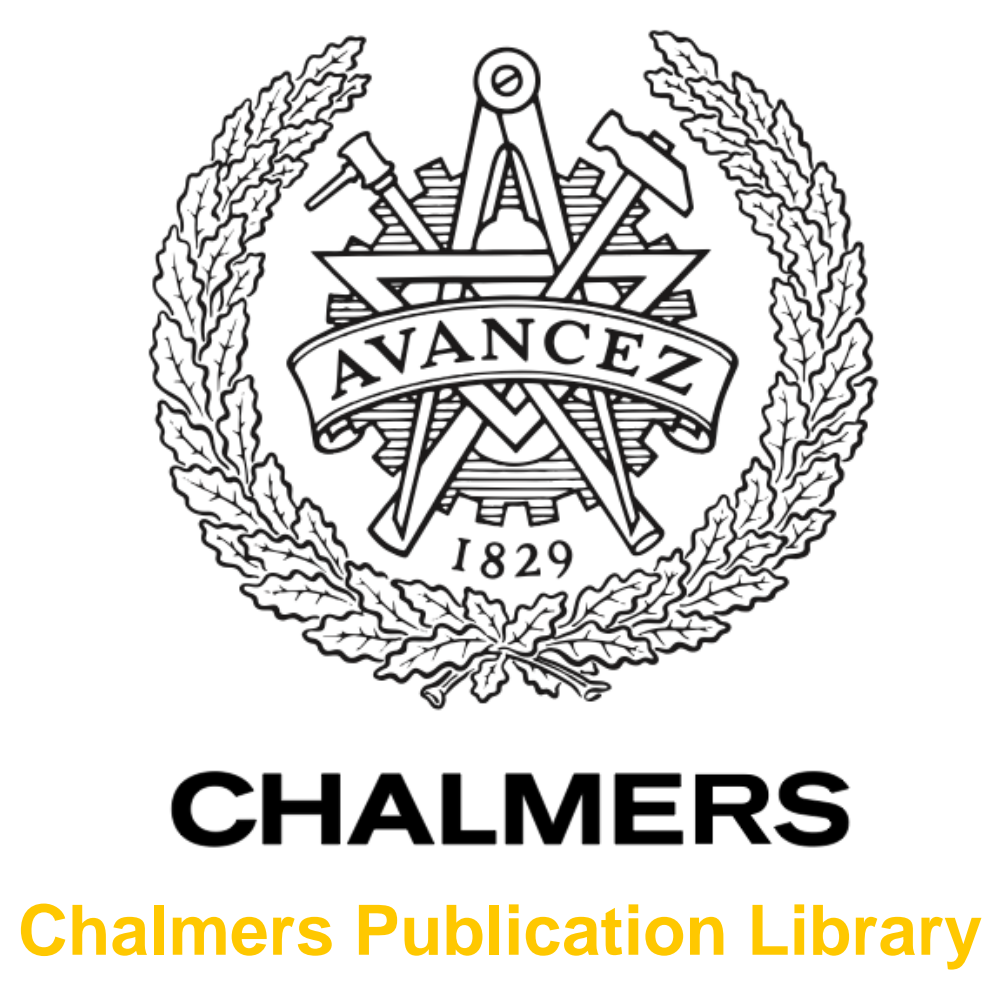

\title{
Microwave imaging of near-field object using ultra-wideband synthetic aperture radar algorithm
}

This document has been downloaded from Chalmers Publication Library $(\mathrm{CPL})$. It is the author's version of a work that was accepted for publication in:

2012 IEEE International Symposium on Antennas and Propagation, Chicago, USA, July 814, 2012 (ISSN: 1522-3965)

Citation for the published paper:

Fayazi, S. ; Lui, H. ; Yang, J. (2012) "Microwave imaging of near-field object using ultrawideband synthetic aperture radar algorithm". 2012 IEEE International Symposium on

Antennas and Propagation, Chicago, USA, July 8-14, 2012

http://dx.doi.org/10.1109/APS.2012.6348657

Downloaded from: http://publications.lib.chalmers.se/publication/164424

Notice: Changes introduced as a result of publishing processes such as copy-editing and formatting may not be reflected in this document. For a definitive version of this work, please refer to the published source. Please note that access to the published version might require a subscription.

Chalmers Publication Library (CPL) offers the possibility of retrieving research publications produced at Chalmers University of Technology. It covers all types of publications: articles, dissertations, licentiate theses, masters theses, conference papers, reports etc. Since 2006 it is the official tool for Chalmers official publication statistics. To ensure that Chalmers research results are disseminated as widely as possible, an Open Access Policy has been adopted.

The CPL service is administrated and maintained by Chalmers Library. 


\section{Microwave Imaging of Near-Field Object Using Ultra-Wideband Synthetic Aperture Radar Algorithm}

\author{
Seyedeh Shaghayegh Fayazi \\ Dept. of Applied Physics and Electronics \\ Umea University of Technology, Umea, Sweden \\ Dept. of Signals and Systems \\ Chalmers University of Technology, Gothenburg, Sweden \\ fayazi@ student.chalmers.se
}

\author{
Hoi-Shun Lui and Jian Yang \\ Dept. of Signals and Systems \\ Chalmers University of Technology \\ Gothenburg, Sweden \\ antony.lui@chalmers.se \\ jian.yang@chalmers.se
}

\begin{abstract}
A simple procedure that suppresses artefact from ultra-wideband (UWB) synthetic aperture radar (SAR) images is proposed and verified using experimental data. The proposed imaging solution is useful for imaging small objects located in the near-field region with less ambiguity.
\end{abstract}

\section{INTRODUCTION}

Ultra-wideband (UWB) technology and its use in imaging and sensing have drawn significant interest in the last two decades. Extensive studies have contributed to utilizing UWB transient scattering for automated target recognition [1] and imaging purposes [2], [3]. In [2] and [3], a UWB synthetic aperture radar (SAR) imaging algorithm was presented and applied for subsurface object imaging. One limitation of the algorithm is that an artefact in the imaging procedure causes ambiguity. In this work, a simple modified procedure is proposed to reduce such artefact. The feasibility of the proposed algorithm is presented and verified using experimental data.

\section{EXPERIMENTAL SETUP}

The UWB imaging system for objects in near-field region is shown in Fig.1. It consists of a self-grounded Bow-Tie antenna [4], [5] located in front of the object, a verticallyplaced rectangular metal plate. To obtain multiple views, the metal plate is placed in 68 equal-spaced positions along a straight line of $1088 \mathrm{~mm}$ long, and the monostatic responses at each of these 68 positions are measured using a network vector analyzer (VNA) from $0.5 \mathrm{GHz}$ to $13 \mathrm{GHz}$. The above setup is equivalent to the case that a single antenna moves along a straight line in front of the static object, and transmits and receives the monostatic responses at these 68 positions, as shown in Fig. 2. Therefore, the SAR algorithm can be employed to have an imaging of the object.

In order to have a high flexible and accurate positioning system for the measurement at a low cost in this study, a straight path of $1088 \mathrm{~mm}$ long was built up of 68 Duplos LEGO blocks, shown in Fig.1. Each Duplos block has a length of $16 \mathrm{~mm}$, and the distance between the antenna and the LEGO path is $715 \mathrm{~mm}$. The entire measurement is performed

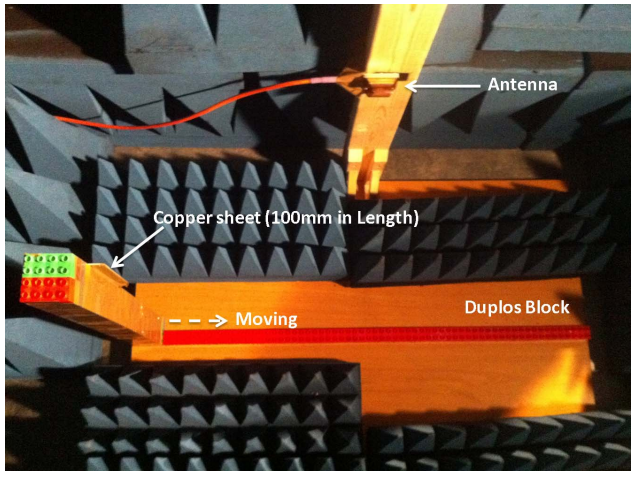

Fig. 1. Experimental setup.

in an anechoic chamber in this preliminary study, so that the reflections from surroundings are minimized. We have developed an algorithm to suppress the reflections from indoor environment and will soon apply it into this imaging project.

The monostatic responses in time domain, which is used for image reconstruction, are obtained via an Inverse Fourier Transform of the frequency samples provided by the VNA measurement.

\section{IMAging Algorithm PRINCIPLE}

An object with a simple geometry, a metal sheet with a length of $100 \mathrm{~mm}$, is considered for the imaging, shown in Fig. 1. When the object is placed at the 68 position, it is equivalent to that the object is located in front of an array of 68 antenna elements, shown in Fig. 2. This setup is similar to those described in [2], [3], except for that only the monostatic responses are measured and used here. The intensity of each pixel $\left.I_{(} m, n\right)$ is given by

$$
I_{(m, n)}=\sum_{k=1}^{K} S_{k}\left(t=T_{k, m, n}\right) .
$$

where $S_{k}$ is the received signal of antenna $k$, and $T_{k, m, n}$ is the round-trip time delay between the $k$ th antenna and the 


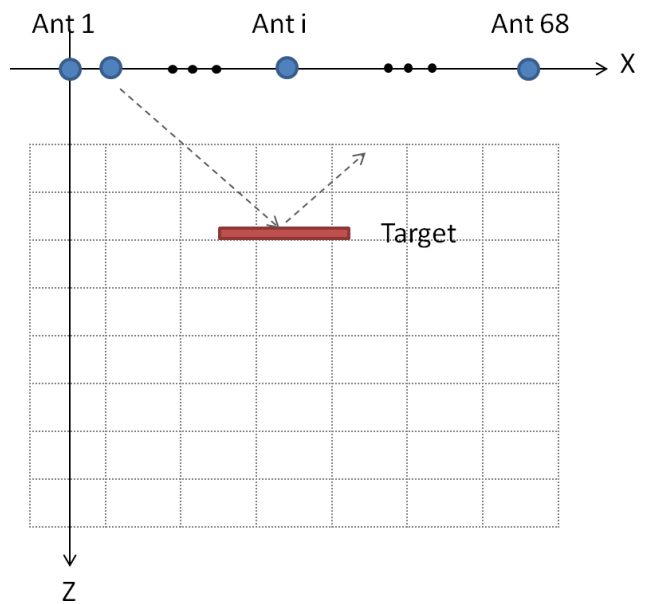

Fig. 2. The geometry of the SAR configuration.

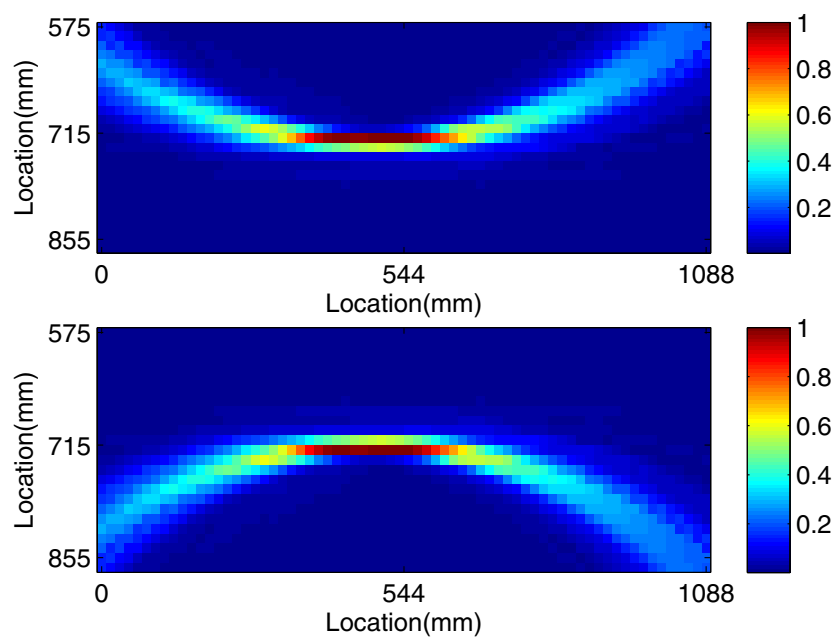

Fig. 3. Image reconstruction results based on: (a) the front antenna (up); (b) the back antenna (down)

physical position of the pixel $(m, n)$.

\section{Modified Algorithm And Results}

The image is reconstructed by using (1) and shown in Fig. 3 (up). The vertically-placed metal plate is observed but it comes with a "smile" shape around it. This smiling is an undesirable artefact introduced from the algorithm. The reason is discussed as follows.

When the image is constructed, the distance $d$ and the round-trip delay $t_{d}=2 d / c$ between the antenna and the pixel are calculated. Then, the signal $S\left(t=t_{d}\right)$ is chosen. If the object is present at this pixel, a strong signal $S\left(t=t_{d}\right)$ is picked up and used for image reconstruction for this pixel. However, the same value is also picked up for other pixels with the same distance $d$ away from the same antenna position. Thus, an artefact arc is formed. In this work, a simple metallic plate is used and the arc forms a "smile" in the resultant image.

To remove this artefact, we use the same antenna moving in the other side of the object, say behind the object. The

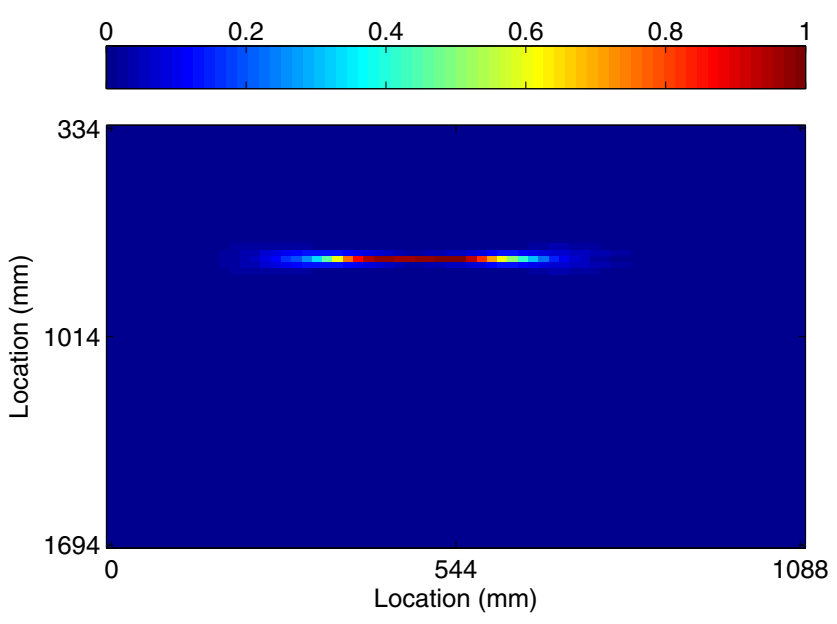

Fig. 4. The resultant image after modified algorithm

same measurement and image reconstruction procedures are repeated. The resultant image is shown in Fig.3 (down). It is observed that the "smile" is changed to a "crying" in the image. By a correlation of these two images - each pixel intensities of the two images are multiplied by each other, the "smile" and the "crying" are canceled while the object image remains. The resultant image is shown in Fig.4 and it is clear that the artefact has been significantly suppressed.

\section{Conclusions}

Imaging of object in the near-field region using a modified UWB-SAR algorithm is presented. The "smile" artefact, which is inherited in the original algorithm, is effectively suppressed using the proposed procedures. The feasibility of the algorithm is demonstrated using experimental data. Future work will focus on better solutions to suppress such artefact using an algorithm for estimating the Direction of Arrival (DOA) of signals.

\section{ACKNOWLEDGEMENT}

This work has been supported by the Swedish research council VINNOVA via a project within the VINN Execellence centre CHASE.

\section{REFERENCES}

[1] H. S. Lui and N. V. Z. Shuley, "Radar Target Recognition using a "Banded" E-Pulse Technique," IEEE Trans. Antennas and Propagat. vol. 54, no. 12, pp. 3874-3881, 2006.

[2] Y. Wang, I. D. Longstaff, and C. J. Leat, "SAR Imaging of Buried Objects from MoM modelled scattered field," IEE Proc. Radar, Sonar and Navigation, vol. 148, no. 3, pp. 167-172, 2001.

[3] S. Vitebskiy, L. Carin, M. A. Ressler, and F. H. Le, "Ultra-Wideband, Short-Pulse Ground-Penetrating Radar: Simulation and Measurement," IEEE Trans. Geoscience and Remote Sensing, vol. 33, no. 3, pp. 762-772, 1997.

[4] J. Yang and A. Kishk, "A novel low-profile compact directional ultrawideband antenna: the self-grounded Bow-Tie antenna," IEEE Trans. Antennas Propagat., vol. 59, accepted 2011.

[5] — "The self-grounded Bow-Tie antenna." Spokane, Washington: 2011 IEEE AP-S International Symp. on Antennas Propag., 3-8 July 2011. 